\title{
Inverse free electron laser accelerator for advanced light sources
}

\author{
J. P. Duris, P. Musumeci, and R. K. Li \\ Department of Physics and Astronomy, UCLA, Los Angeles, California 90095, USA
}

(Received 25 October 2011; published 12 June 2012)

\begin{abstract}
We discuss the inverse free electron laser (IFEL) scheme as a compact high gradient accelerator solution for driving advanced light sources such as a soft x-ray free electron laser amplifier or an inverse Compton scattering based gamma-ray source. In particular, we present a series of new developments aimed at improving the design of future IFEL accelerators. These include a new procedure to optimize the choice of the undulator tapering, a new concept for prebunching which greatly improves the fraction of trapped particles and the final energy spread, and a self-consistent study of beam loading effects which leads to an energy-efficient high laser-to-beam power conversion.
\end{abstract}

DOI: 10.1103/PhysRevSTAB.15.061301

PACS numbers: 41.75.Jv, 41.75.Lx, 41.60.Cr

\section{INTRODUCTION}

The interaction of an electron beam and a laser within an undulator is the subject of numerous studies in recent accelerator and beam physics as it allows particle accelerators to benefit from the tremendous progress achieved by laser technology. Aside from the free electron laser (FEL) amplification, applications of this interaction include beam microbunching at radiation scale [1], control of intrinsic energy spread by laser heating [2], optical stochastic cooling [3], bunch train phase locking [4], and many others $[5,6]$. One particular application which is the subject of this paper is laser-driven particle acceleration. In this case, energy is extracted from the radiation and transferred to the electrons, and it is customary to refer to the system as an inverse free electron laser (IFEL).

Use of the IFEL acceleration scheme to accelerate particles was suggested back in 1972 by Palmer [7] and then reconsidered as a high gradient solution for high-energy physics applications by Courant, Pellegrini, and Zakowicz in 1985 [8]. Recent experiments have shown that IFEL can achieve both very high-energy gradient and relatively good output beam quality $[9,10]$.

Other significant advantages over different advanced accelerator schemes include the fact that IFEL, being a vacuum far-field acceleration scheme, does not require any medium (plasma or dielectric) or boundaries close to the interaction. Because of its relative simplicity, the IFEL has matured as one of the most reliable advanced accelerator techniques. In fact, fundamental steps in laser-based acceleration research such as the demonstration of staging and production of monoenergetic beams have been obtained first using the IFEL scheme [9]. The major drawback of this technique is that, due to synchrotron radiation losses,

Published by the American Physical Society under the terms of the Creative Commons Attribution 3.0 License. Further distribution of this work must maintain attribution to the author(s) and the published article's title, journal citation, and DOI. the final energy of an IFEL accelerator is limited to a few $\mathrm{GeV}$ [8]. This has been a show stopper for further development of IFEL accelerators for high-energy physics accelerators.

On the other hand, there are a number of applications which demand compact 1-2 GeV energy high quality electron beams. The IFEL is perfectly suited as a driver for applications like soft $\mathrm{x}$-ray free electron laser amplification [11] and the production of gamma rays by inverse Compton scattering (ICS) [12]. In the latter case, a significant advantage comes from the fact that the high power laser system for the ICS source can be shared and used also to drive the IFEL accelerator.

Driven by these applications, the interest in IFEL accelerators has sparked again in recent years with various experiments planned to demonstrate the extension of the IFEL to the $100 \mathrm{MeV}$ energy range [13,14]. The goal of this paper is to provide a modern background for the design of high gradients IFEL accelerators for advanced light sources and to discuss recent advancements that can largely improve the characteristics of future IFEL's. For light source applications, the output beam quality is very important. In particular, the preservation of the input emittance and the control of the final energy spread are IFEL attractive attributes.

The paper is organized as follows. In the next section we will describe the IFEL accelerator scheme and give general design guidelines for a single stage high-energy high gradient IFEL module. Then we will present a procedure to determine the optimum undulator tapering. A novel IFEL prebuncher scheme will be discussed in Sec. IV, and finally in the last section we will show the results of selfconsistent IFEL simulations where the effects of beam loading have been included in the simulations.

\section{HIGH GRADIENT IFEL ACCELERATOR LAYOUT}

The traditional treatment of the IFEL interaction (see for example [8]) considers an electromagnetic monochromatic 
wave of frequency $\omega=k c$ copropagating with electrons oscillating in an undulator with period $\lambda_{w}=2 \pi / k_{w}$ and magnetic field amplitude $B_{0}$. It is customary to introduce the normalized amplitude of the undulator vector potential $K=e B_{0} / m_{0} c k_{w}$. Assuming that there is no feedback on the radiation from the particle motion (we will come back extensively to this point in the last section of this paper), the 1D particle dynamics can be well described by the period-averaged equations,

$$
\begin{aligned}
& \frac{d \gamma}{d z}=\frac{k K_{l} K}{2 \gamma} \mathrm{JJ} \sin \theta \\
& \frac{d \theta}{d z}=k_{w}-k \frac{1+K^{2} / 2}{2 \gamma^{2}},
\end{aligned}
$$

where $\gamma$ is the normalized particle energy and $\theta=k_{w} z+$ $k z-\omega t$ is the ponderomotive phase-that is the phase of the particles in the cos-like potential formed by the combined action of the laser and the undulator field. $K_{l}=$ $e E_{0} / m_{0} c^{2} k$ is the normalized vector potential of the electromagnetic wave. We assume $K_{l} \ll K$ in this paper. JJ $=$ $\left[J_{0}(G)-J_{1}(G)\right]$ with $G \simeq \frac{K^{2}}{4+2 K^{2}}$ is the Bessel function factor which takes into account the reduction in the energy exchange rate in a planar undulator. For large $K$, JJ $\rightarrow 0.7$.

For the helical undulator (and a circularly polarized laser beam), the equations of the IFEL interaction simplify due to the absence of oscillatory terms in the particle longitudinal velocity, and we have

$$
\begin{aligned}
& \frac{d \gamma}{d z}=\frac{k K_{l} K}{\gamma} \sin \theta \\
& \frac{d \theta}{d z}=k_{w}-k \frac{1+K^{2}}{2 \gamma^{2}} .
\end{aligned}
$$

The main differences when comparing the accelerating gradient equations in the helical and planar geometry are the Bessel function factor and the extra factor of 2 in the denominator for the planar case. For a fair comparison, it is important to note though that, for a fixed laser power available, the peak electric field (and hence $K_{l}$ ) in the helical interaction geometry is $\sqrt{2}$ times smaller due to the laser circular polarization. Taking this into account, the advantage of helical undulator is a $\sim 2$ larger gradient than in the planar undulator case. Because of this, in the rest of this paper we will restrict ourselves to the helical IFEL interaction geometry.

If the relative phase between the particles and the radiation oscillates rapidly, no net energy exchange occurs. Efficient interaction occurs only when the variation of the ponderomotive phase is minimized. It is useful then to introduce the synchronous or resonant particle for which the resonant condition

$$
\gamma_{r}=\sqrt{k \frac{1+K^{2}}{2 k_{w}}}
$$

is satisfied, the phase $\theta_{r}$ is constant, and significant energy exchange takes place [15]. The energy of the resonant particle varies according to

$$
\frac{d \gamma_{r}}{d z}=\frac{k K_{l} K}{\gamma_{r}} \sin \theta_{r}
$$

Even neglecting beam loading, the electromagnetic field amplitude is constant along the undulator only in the case of an ideal plane wave. A better approximation consists of describing the electromagnetic wave with a complex amplitude $E(z)=E_{0}(z) e^{i \phi(z)}$ varying along the undulator. This can be introduced in the IFEL model by a $z$-dependent $K_{l}(z)$ and introducing in the phase equation a term $d \phi / d z$ which takes into account the variation of the radiation phase along the undulator.

Most of the IFEL experiments carried out so far have simply used a freely propagating laser wave in vacuum. Optical waveguides or refocusing schemes have been considered as they offer the possibility of maintaining the highest possible laser intensity all along the interaction length $[16,17]$. On the other hand, efficient coupling and guiding of high power laser pulses for relatively large distances has not been achieved yet. Further, a waveguide or a series of lenses spoils one of the significant advantages of a far-field accelerator which consists in being free of damage threshold limitations. Another important point to notice is that nowadays high power laser systems can easily provide multi-TW laser intensities which, as we will see, are large enough to allow reaching $1-2 \mathrm{GeV}$ energies in one stage, avoiding the complications of cascading multiple accelerating modules or refocusing the laser. For these reasons, in our treatment of the IFEL accelerator as an advanced light source driver, we can restrict our analysis to a single IFEL stage driven by a freely propagating diffracting high power laser pulse.

As a first approximation, we can model the laser beam as the fundamental Gaussian mode $\mathrm{TEM}_{00}$ with focal position $z_{w}$ and Rayleigh range $z_{r}=\pi w_{0}^{2} / \lambda$, where $w_{0}$ is the $1 / e^{2}$ focal spot size. The electric field in the focus for circular polarization can be calculated from the input power $E_{0}=$ $\sqrt{Z_{0} P / \pi w_{0}^{2}}$, where $Z_{0}=377 \Omega$ is the vacuum impedance. The normalized field amplitude along the undulator can be written as

$$
K_{l}(z)=\frac{e_{0} E_{0}}{m_{0} c^{2} k} \frac{w_{0}}{w(z)} \propto \sqrt{\frac{1}{z_{r}} \frac{1}{1+\left(\frac{z-z_{w}}{z_{r}}\right)^{2}}} .
$$

The phase of the electromagnetic wave presents the wellknown Gouy phase shift so that an extra term $d \phi / d z=$ $1 /\left(1+\left(z-z_{w}\right)^{2} / z_{r}^{2}\right)$ appears in the equation for the evolution of the ponderomotive phase, Eq. (2).

In order to maximize the energy exchange, it is natural to choose the focal plane position $z_{w}=L_{u} / 2$ halfway through the interaction region. The choice of the spot 


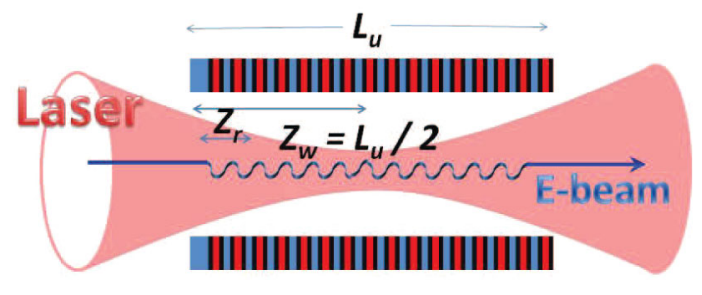

FIG. 1. Scheme for a diffraction dominated inverse free electron laser accelerator.

size and related Rayleigh range requires a deeper analysis. The optimum point is a result of a compromise between (a) focusing tighter to maximize the intensity at the focal plane and (b) keeping the beam size and radiation intensity uniform along the undulator in order to increase the effective acceleration length. The scheme is illustrated in Fig. 1.

We can find such an optimum by maximizing the integral of the accelerating gradient along the undulator. Integrating Eq. (4), we obtain

$$
\Delta\left(\gamma_{r}^{2}\right)=\int_{0}^{L_{u}} 2 k K K_{l}(z) \sin \theta_{r} d z .
$$

For an IFEL design with a constant $K$ and constant $\theta_{r}$ along the undulator, we can bring all the constants out of the integral, and the optimum Rayleigh range is found by using the condition

$$
\frac{\partial}{\partial z_{r}}\left[\int_{0}^{L_{u}} \sqrt{\frac{1}{z_{r}} \frac{1}{1+\left(\frac{z^{-\frac{L_{u}}{2}}}{z_{r}}\right)^{2}}} d z\right]=0
$$

whose solution (numerically obtained) is $\frac{z_{r}}{L_{u}}=0.15$.

In the more general case, the undulator is tapered to achieve a large energy gain, and both $K$ and $\sin \theta_{r}$ can change (increase) along the interaction. We can take this into account by solving Eq. (7) allowing in the integral a $z$-dependent term. We show in Fig. 2 the solution of Eq. (7) for different functions varying as $z^{n}$. Even for a relatively strong variation of the integrand $(n>4)$, the maximum gradient is a relatively slowly varying function of the ratio $z_{r} / L_{u}$ and the optimum shifts to values closer to 0.3 (see Fig. 2). The conclusion is that, for given input power $P$, the best coupling of a diffraction dominated laser beam and an electron beam in an IFEL is obtained for $0.15<$ $z_{r} / L_{u}<0.3$, weakly dependent on the undulator tapering. In the rest of this paper we will consider $z_{r} / L_{u} \sim 0.2$.

Using this result and Eq. (6), it is possible to obtain an estimate for the IFEL energy gain with a given laser power available,

$$
\begin{aligned}
\Delta\left(\gamma^{2}\right) & =2 \sqrt{2} \frac{e_{0} \bar{K}}{m_{0} c^{2}} \sqrt{\frac{Z_{0} P z_{r}}{\lambda}} \int_{0}^{L_{u} / 2 z_{r}} \sqrt{\frac{1}{1+x^{2}}} d x \\
& \cong \frac{8}{\sqrt{5}} \bar{K} \sqrt{P / P_{0}} \sqrt{L_{u} / \lambda},
\end{aligned}
$$
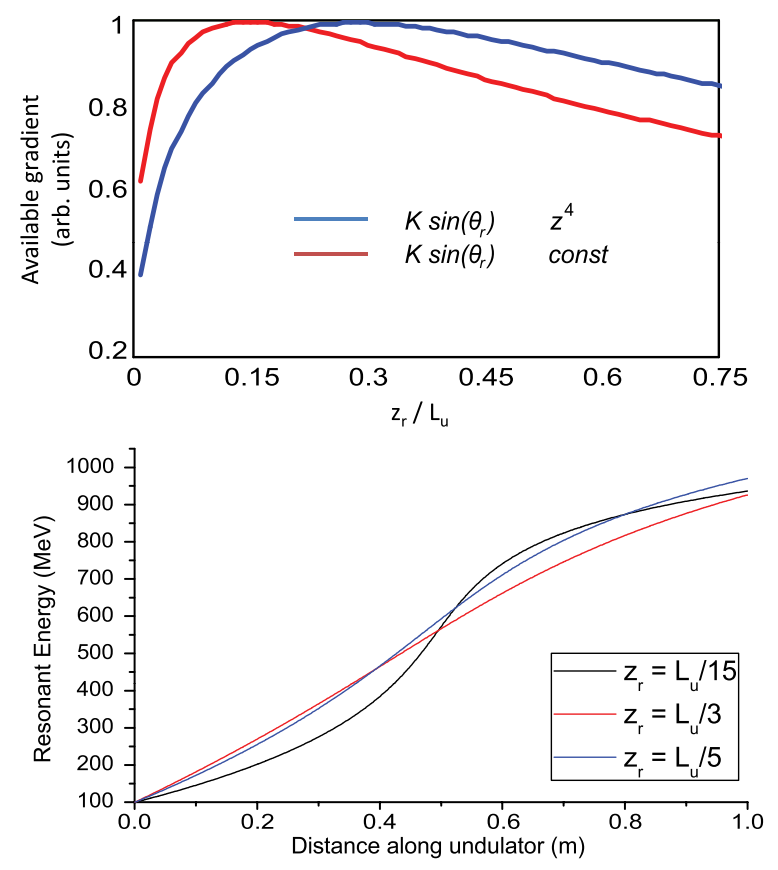

FIG. 2. Left: Available gradient vs normalized Rayleigh range for two different tapering schemes. Right: Resonant energy as a function of undulator length for three different ratios $z_{r} / L_{u}$. The maximum final energy is achieved for a Rayleigh range of $L_{u} / 5$.

where $P_{0}=m_{0}^{2} c^{4} / e_{0}^{2}, Z_{0}=693 \mathrm{MW}$, and we have assumed a constant resonant phase $\theta_{r}=\pi / 4$.

The beam's final energy is $\gamma_{f}=\sqrt{\gamma_{0}^{2}+\Delta\left(\gamma^{2}\right)}$; however, for a total energy gain much larger than the initial energy, we can neglect $\gamma_{0}$ and approximate the final energy as $\gamma_{f}=\sqrt{\Delta\left(\gamma^{2}\right)}$. An important point we get from Eq. (8) is that the final energy only goes like $P^{1 / 4}$ and $L_{u}^{1 / 4}$. So in order to double the final energy, we need to have 16 times larger laser power or longer undulator. This rather poor scaling law is a consequence of the fact that the IFEL is a second order acceleration mechanism while the final energy is proportional to the square root of the available electric field. $\bar{K}$ is the averaged normalized amplitude along the undulator. As we will see in the next section, $K$ actually varies along the undulator, but it usually has a value between 1 and 10. In order to get a practical estimate for the laser power required to drive an IFEL for advanced light sources application, let us assume $\bar{K}=5$. Using $0.8 \mu \mathrm{m}$ radiation and a $1 \mathrm{~m}$ long undulator, the power required to get a $1 \mathrm{GeV}$ output beam is $\sim 20 \mathrm{TW}$. These power levels are available using compact commercial laser systems [18].

\section{TAPERING OPTIMIZATION}

The problem of designing an IFEL accelerator consists of optimizing the output beam parameters by simultaneously varying the undulator period $\lambda_{w}(z)$ and magnetic field amplitude $B(z)$ along the interaction. The rate of 
change of the resonant energy described by Eq. (4) implies the existence of a maximum available ponderomotive gradient given by a value of the resonant phase $\theta_{r} \cong \pi / 2$. In practice, the choice of the resonant phase $\theta_{r}$ has an important effect on the performances of the accelerator. For $\theta_{r}=0$ there is no acceleration and the system acts as a buncher. The rate of acceleration is the largest when $\theta_{r}=\pi / 2$, but to obtain a stable acceleration for nonresonant particles for a larger area in the longitudinal phase space, one is forced to choose a lower resonant phase and have a smaller acceleration rate. A good compromise is usually obtained by choosing $\theta_{r}=\pi / 4$.

To obtain a constraint on the possible variations of $\lambda_{w}(z)$ and $B(z)$ along the undulator, we shall require the rate of increase in the resonant energy due to the tapering of the period and magnetic field amplitude to match the available ponderomotive gradient. In other words,

$$
\left[\frac{d\left(\gamma_{r}^{2}\right)}{d z}\right]_{\text {tapering }}=\left[\frac{d\left(\gamma_{r}^{2}\right)}{d z}\right]_{\text {ponderomotive }},
$$

where the left side is obtained deriving Eq. (3) and the right side is obtained rearranging Eq. (4).

Imposing only this condition is not sufficient to uniquely define the two unknown functions $\lambda(z)$ and $B(z)$. There is an additional degree of freedom in choosing how to vary the tapering along the undulator length. In [8] different solutions for tapering the undulator have been considered. In particular, the authors analyzed undulator tapering with constant period $\lambda_{w}$, constant magnetic field amplitude $B$, or constant undulator parameter $K$. In practice, though, a given undulator design will be characterized by a fixed relationship between the magnetic field amplitude and the wiggling period [i.e. $B(z)=B\left(\lambda_{w}(z)\right)$ ]. Such an undulatorbuilder equation can be used as the other constraint to close the system.

A single differential equation for $\lambda_{w}(z)$ can then be obtained from Eq. (9):

$$
\frac{d \lambda_{w}}{d z}=\frac{8 \pi K_{l}(z) K\left(\lambda_{w}\right) \sin \theta_{r}}{1+K\left(\lambda_{w}\right)^{2}+2 \lambda_{w} K\left(\lambda_{w}\right) \frac{\partial K\left(\lambda_{w}\right)}{\partial \lambda_{w}}}
$$

whose solution yields the optimal variation of the undulator period along the IFEL accelerator. Once $\lambda_{w}(z)$ is known, the magnetic field amplitude variation immediately follows by using the undulator-builder equation. The initial value for this first order differential equation, the period length at the beginning of the undulator, is defined by imposing the resonance condition at the input beam energy. The term $\frac{\partial K}{\partial \lambda_{w}}$ can be derived from the undulator-builder equation and in general will depend on the details of the magnetic and mechanical design of the undulator, i.e., if the undulator is an electromagnet, or a permanent magnet one, the undulator gap, the characteristics of the permanent magnet material, and so on.
A very effective way to obtain a helical undulator field is to use the Halbach geometry for permanent magnet undulators with two undulator magnet arrays rotated by 90 degrees and shifted by $\pi / 2$ in phase [19]. For a pure Halbach undulator, the undulator-builder equation or the relation between the magnetic field amplitude $B$ and the period length $\lambda_{w}$ for a given gap $g$ and magnet length $L_{m}$ is

$$
B=1.8 B_{r} e^{-\pi g / \lambda_{w}}\left(1-e^{-2 \pi L_{m} / \lambda_{w}}\right) .
$$

In Fig. 3, the solutions of Eq. (10) when $\partial K / \partial \lambda_{w}$ is calculated using the above undulator-builder equation are shown. For these calculations we have assumed a Ti:Sa laser $(\lambda=0.8 \mu \mathrm{m})$ with an input power of $20 \mathrm{TW}$, a $1 \mathrm{~m}$ long undulator, and an input energy of $100 \mathrm{MeV}$. The different curves correspond to different parameters of a helical Halbach permanent magnet undulator.

The results of the tapering design optimization are verified using a three-dimensional simulation code which has been benchmarked against the results of the UCLA and BNL IFEL experiments. The code tracks the evolution of the particles by solving the Lorentz force equation in the combined field of an (analytically given) laser field and the undulator magnetic field profile generated by the optimization routine. It includes the effect of the undulator focusing. A summary of the parameters used in the simulations is listed in Table I.



FIG. 3. (a) Resonant energy along the undulator for different cases. (b) Variation of undulator period $\lambda_{w}$ along the undulator. (c) Variation of magnetic field amplitude $B$ along the undulator. 
TABLE I. Inverse free electron laser accelerator parameters.

\begin{tabular}{lc}
\hline \hline Electron beam & \\
\hline Input energy & $100 \mathrm{MeV}$ \\
Emittance & $0.25 \mathrm{~mm} \mathrm{mrad}$ \\
Peak current & $100 \mathrm{Amp}$ \\
$\sigma_{\text {rms }}$ at focus & $100 \mu \mathrm{m}$ \\
\hline Laser & \\
\hline Power & $20 \mathrm{TW}$ \\
Wavelength & $0.8 \mu \mathrm{m}$ \\
Spot size $\left(1 / \mathrm{e}^{2}\right)$ at focus & $240 \mu \mathrm{m}$ \\
Rayleigh range & $20 \mathrm{~cm}$ \\
Undulator length & $1 \mathrm{~m}$ \\
\hline \hline
\end{tabular}

The first solution presented refers to the use of relatively cheap and durable $\mathrm{NdFeB}$ magnets $\left(B_{r}=1.22 \mathrm{~T}\right)$ as permanent magnets. The final IFEL output energy in this case is $0.91 \mathrm{GeV}$ with a $0.8 \mathrm{GV} / \mathrm{m}$ average accelerating gradient. Recently praseodymium based undulators with residual magnetization $B_{r}$ up to $1.7 \mathrm{~T}$ have been proposed [20,21]. As evident from Fig. 3, this option is particularly interesting for IFEL accelerators as it allows a considerable gain in final output energy.

The main requirement for the gap $g$ is that the clear aperture between the magnet is sufficiently large to allow full clearance for the beam and the electromagnetic radiation transport. The latter usually results in a more stringent requirement since the geometric electron beam emittance is usually smaller than the equivalent laser beam emittance $\lambda / 4 \pi$. This translates to a gap at least $\pi$ times larger than the Gaussian laser beam size $w(z)$. It is possible to select a design with a gap constant or varying along the undulator. By allowing the gap to vary along the undulator proportionally to the laser beam profile, one could increase the magnetic field amplitude right where the intensity of the laser is larger and so a slightly higher final output energy is achieved. In Fig. 3 we show the case of a constant gap $g=5 \mathrm{~mm}$ and the case where we allow the gap to vary from $g=5 \mathrm{~mm}$ at the undulator entrance and exit to $g<3 \mathrm{~mm}$ in the middle of the undulator at the laser focus.

Finally, so far we have assumed a resonant phase $\theta_{r}=$ $\pi / 4$ constant along the undulator. A very interesting possibility is to vary the resonant phase along the IFEL accelerator. A resonant phase closer to $\pi / 2$ increases the gradient, but reduces the phase space area of the accelerating bucket and, hence, the stability of the accelerator. Liouville's theorem forbids any attempt of decreasing the phase space area occupied by the beam. On the other hand, the longitudinal phase space area of the trapped bucket is proportional to $\gamma_{r}$ which is increasing along the IFEL undulator. Hence, it is possible to increase the resonant phase along the IFEL without any losses of trapped particles. There are significant advantages in doing this. At the beginning, a resonant phase $\theta_{r} \cong 0$ eases trapping and capture of unbunched electron beams. A larger resonant phase at the end of the undulator maximizes the final energy and even more importantly minimizes the final energy spread. This feature is very important especially for FEL light source applications which require an energy spread smaller than $10^{-3}$. In practice, the initial phase space area occupied by the beam is very small, much smaller than the area of the ponderomotive bucket, due to the very low slice energy spread of rf photoinjector beams, so even taking into account a small longitudinal emittance growth along the acceleration, a final resonant phase close to $\pi / 2$ is possible.

In Fig. 4 we show the longitudinal phase spaces for the two cases of constant and varying $\theta_{r}$. In the second case, the resonant phase is increased linearly after the undulator midpoint to a final value $\theta_{r}=\pi / 2$. The fraction of particles trapped in the bucket and accelerated to the final energy is in both cases around $40 \%$. It is noticeable that, for the varying resonant phase case, the final energy is higher by $>100 \mathrm{MeV}$ and the energy spread is reduced by a factor of 2 to $0.5 \%$. This is particularly important for advanced light source applications where the beam energy spread is a sensitive parameter as, for example, in the determination of the FEL gain length.
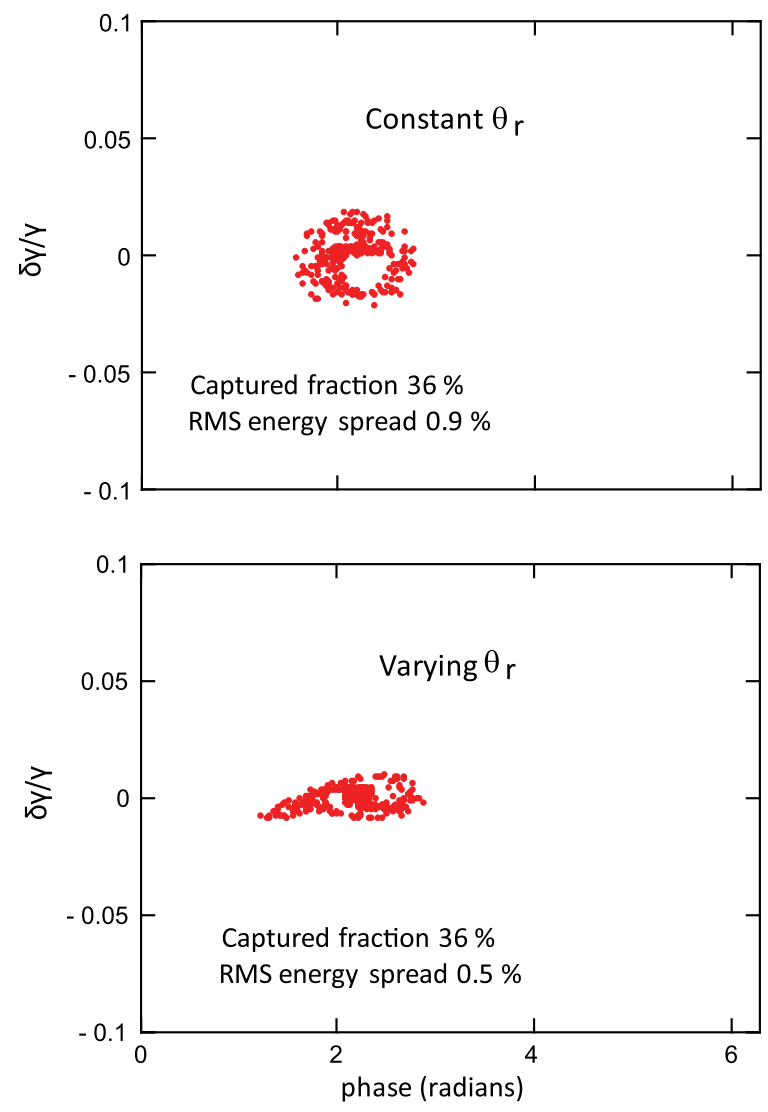

FIG. 4. Longitudinal phase space at the end of the undulator for the constant resonant phase and for the varying resonant phase undulator. 
It should be noted that, according to the 3D simulations, the normalized transverse emittance is essentially constant along the interaction growing by less than $0.1 \mathrm{~mm} \mathrm{mrad}$ from $0.25 \mathrm{~mm} \mathrm{mrad}$ at the undulator entrance to $0.33 \mathrm{~mm}$ mrad at the exit. So production of extremely bright highenergy beams is possible with an IFEL accelerator.

A study of the overlap of the laser and particle transverse profiles showed that the final energy, relative energy spread, and emittance remain constant while the accelerated fraction of the beam decreases as the transverse size of the beam is increased incrementally from $50 \mu \mathrm{m}$ to $1 \mathrm{~mm}$. The final rms transverse size of the accelerated bunch also remains constant reflecting the fact that only the part of the beam in the area of the laser with sufficient intensity is accelerated. Temporal overlap inefficiencies were not accounted for.

\section{IFEL PREBUNCHING}

Using an IFEL prebuncher to modulate the beam density at the scale of the optical wavelength prior to injection into the IFEL accelerator can greatly improve the output beam quality of the accelerator. In an IFEL prebuncher a relatively low power laser beam copropagates with a relativistic electron beam in a short nontapered undulator. Because of the cos-like ponderomotive potential, the electron beam performs synchrotron oscillations in longitudinal phase space. If the laser power is such that the end of the undulator corresponds to $1 / 4$ synchrotron period, then a beam microbunched at the laser wavelength is obtained. Most often, a short dispersive section with $R_{56}>0$ is used to convert the energy modulation resulting from the IFEL interaction into density modulation. The laser pulse driving the prebuncher is typically obtained from the main laser driving the accelerator so that the resulting microbunches are synchronized and phase locked with the laser wave.

Particular care has to be taken to preserve the microbunching from the buncher to the accelerator, but recent experiments both at $10.6 \mu \mathrm{m}$ [9] and $800 \mathrm{~nm}$ [4] have confirmed that it is in fact possible to inject a beam into a laser-driven accelerating structure with a narrow phase spread.

The maximum density modulation that can be achieved with an IFEL prebuncher is set by the nonlinearities of the pendulum like IFEL dynamics and the minimum microbunch rms size is typically $1 / 10$ th of the laser wavelength. The bunching factor $B=\left|\sum_{i} e^{i k z_{i}}\right| / N$, where $z_{i}$ are the particle longitudinal positions and $N$ is the number of particles, is another parameter that can be used to quantify the quality of the microbunching at a given wave number $k$. Even for the ideal case of a vanishing uncorrelated energy spread for the input beam, $B$ is still limited by the peak of the first order Bessel function and reaches maximum values around $\cong 0.6$.

A possible improvement to this prebunching scheme comes from taking advantage of the harmonic IFEL interaction in planar undulators and modifying the IFEL ponderomotive potential in order to reduce the nonlinearities and achieve almost perfect bunching. It is well known that, due to the modulation of the longitudinal velocity, electrons in a planar undulator interact not only with the fundamental laser wavelength but also with its harmonics. The coupling coefficient has been calculated theoretically and verified experimentally [22-24]:

$$
\begin{aligned}
\mathrm{JJ}_{n}^{*}= & (-1)^{(n-1) / 2}\left[J_{(n-1) / 2}(G)-J_{(n+1) / 2}(G)\right] \text { for } n \text { odd } \\
& (-1)^{(n+1) / 2} \xi\left[J_{(n-2) / 2}(G)-J_{(n+2) / 2}(G)\right] \text { for } n \text { even, }
\end{aligned}
$$

where $\xi=k K \phi / \gamma k_{w}$. For the even harmonics, the on-axis interaction is suppressed and the coupling is proportional to the angle $\phi$ between the laser beam and the beam trajectory prior to entering the undulator. Since any function can be expanded in its Fourier series, it is in principle possible to fully control the shape of the ponderomotive potential and the evolution of the electron distribution by properly synthesizing the electromagnetic wave driving the prebuncher, and $a d$ hoc tuning the interaction coefficients.

Initial theoretical studies of multicolor IFEL prebunchers had already shown promising results in terms of bunching enhancement [25]. Here we analyze the practical case when the IFEL is driven by the first four harmonics of the fundamental laser frequency with optimized amplitudes and phases to generate a nearly ideal parabolic potential with a linear restoring force. In this case the dynamical evolution of this system resembles more the one of a harmonic oscillator than the one of a physical pendulum. An ideal parabolic potential function $\left(\propto x^{2}\right)$ can be Fourier expanded as $\sum_{n} a_{n} \cos (n x)$, where $a_{n} \propto 1 / n^{2}$ are the coefficients of the expansion. Since it is experimentally challenging to generate a driving laser wave consisting of more than a few spectral lines, in the implementation of our scheme we choose to truncate the series at $n=4$ still obtaining a very good approximation of the harmonic potential (see Fig. 5).

Let us consider a prebuncher driven by a $800 \mathrm{~nm}$ laser pulse. Frequency multiplication in a nonlinear optical crystal can easily yield up to the 4th harmonic of a Ti:Sa laser. The different colors of the radiation are phase locked due to the generation process and optical flats with different phase advance for the different wavelengths can be used to control the relative phases of the four laser lines at the undulator entrance. Full control on the phase of each of the harmonics of the laser is required in order to control the shape of the optical waveform and hence of the interaction potential. Synthesis of ultrafast shaped waveforms from five discrete optical harmonics has already been demonstrated experimentally by a few groups [26,27]. Our proposed scheme is shown in Fig. 6.

An undulator with a relatively large $K$ enhances the harmonic coupling and minimizes the laser power required to drive the bunching. For simplicity, we take a $40 \mathrm{~cm}$ long 


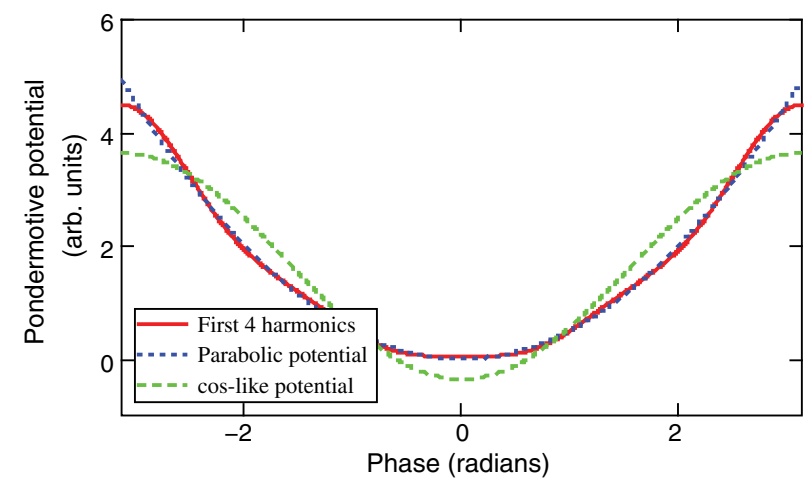

FIG. 5. Ponderomotive pendulum like potential. Harmonic oscillator potential and approximation with first 4 harmonics.

undulator with period $\lambda_{w}=2.5 \mathrm{~cm}$. The electron beam energy is chosen to be at the injection energy of the IFEL high-energy laser accelerator discussed in the previous section of this paper. In order to have a resonant condition with the $800 \mathrm{~nm}$ fundamental laser wavelength, we choose $K=1.84$. The coupling with the even harmonics is obtained by injecting at an angle $\theta=0.7 \mathrm{mrad}$ the radiation and the particles in the undulator which yields $\xi=0.2$ in Eq. (12). To maintain the overlap of the beam with the laser over the entire undulator distance, we choose a relaxed focusing condition $z_{r}=L_{u}$ which yields a laser focal spot size $w_{0}=320 \mu \mathrm{m}$. A larger injection angle $\theta$ would maximize the even harmonic coupling, but it would also require to increase the laser spot size (which for a given laser power lowers the available intensity) to ensure a good spatial overlap between the particles and the laser throughout the $40 \mathrm{~cm}$ long undulator.

By dividing the coefficients $a_{n}$ for corresponding coupling factors $\mathrm{JJ}_{n}$, one obtains the normalized laser field amplitudes at the different frequencies which are needed to approximate the ideal harmonic oscillator potential. Considering a spot area $\pi w_{0}^{2} / 2=0.16 \mathrm{~mm}^{2}$ and assuming a $100 \mathrm{fs}$ pulse length, this yields the energies per pulse reported in the scheme in Fig. 6.

In order to verify our expectations, we have modified our three-dimensional IFEL code to include the option of using a multicolor laser wave to drive the interaction in the undulator. The result of the simulation is shown in Fig. 7(a) where the longitudinal phase space at the end of the undulator is compared to the case when only the fundamental laser wavelength is used. The residual nonlinearities are the source of the zigzag shape of the longitudinal phase space and are due to the truncation of the Fourier series. Nevertheless, the four harmonic buncher scheme yields more particles bunched in a narrow phase spread around $\theta_{r}=0$ than the classical scheme which shows the characteristic S-shape of the physical pendulum dynamics.

In Fig. 7(b) we show the evolution of the bunching factor induced by a simple single frequency component (traditional prebuncher) and the one induced by our properly tuned frequency combination. Also in this case the difference is significant and a bunching factor $B=0.95$ is obtained.

We performed simulations to verify the stability of the proposed scheme against fluctuations in power and/or phase for each of the four laser harmonics. We ran 1000 different simulations where we allowed $10 \%$ random amplitude fluctuations (corresponding to $20 \%$ power variation), and added a random 0.5 rad phase jitter on each of the laser lines. State of the art optical waveform synthesis experiments have already demonstrated better than few per cent amplitude control and phase jitters smaller than $0.05 \pi$ [26], so our assumptions are reasonable. The histogram of the bunching factor at the end of the prebuncher is displayed in Fig. 8 showing excellent stability in the performances of the harmonic prebuncher with $90 \%$ of the shots having bunching factor above 0.9 .

Even though with a multicolor prebuncher it is possible to pack more particles in the useful acceptance area of the accelerator and improve significantly its performances, it is undeniable that the scheme is significantly more complex than a simple IFEL prebuncher. Such complexity probably offsets the advantages of its implementation in systems aimed at radiation production enhancement. The increase in the coherent energy radiated out by the bunch at a given wavelength is proportional to the square of the bunching

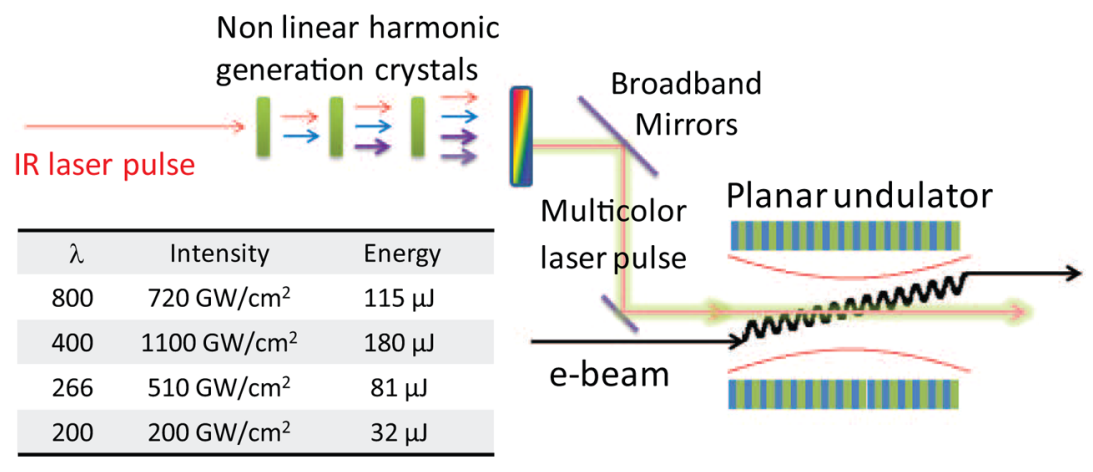

FIG. 6. Scheme of a nearly ideal harmonic prebuncher which uses nonlinear conversion crystals to generate up to the fourth harmonic of an infrared laser driver. 

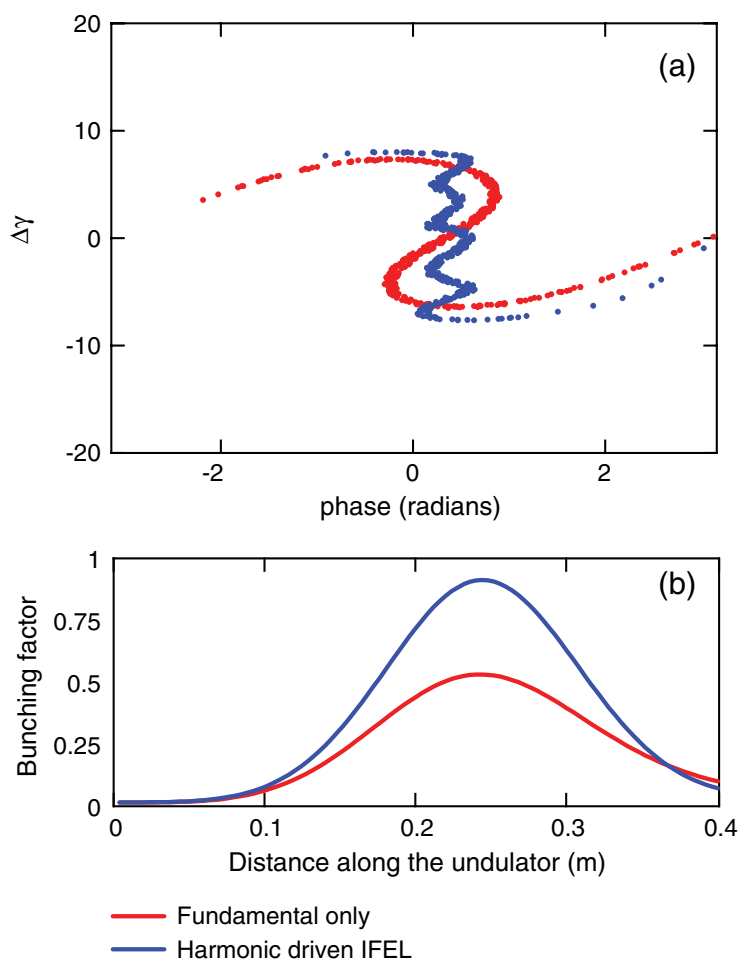

FIG. 7. Longitudinal phase space and longitudinal distribution of a typical and a harmonic prebuncher.

factor. Microbunching the beam using 4 different laser harmonics only adds at most an extra factor of 2 to the output radiation power at the fundamental wavelength. On the other hand, for high harmonic generation schemes, harmonic microbunching has been suggested as a way to significantly increase the radiation output [28]. In the context of this paper, application of the harmonic IFEL microbunching scheme as injector for high frequency high

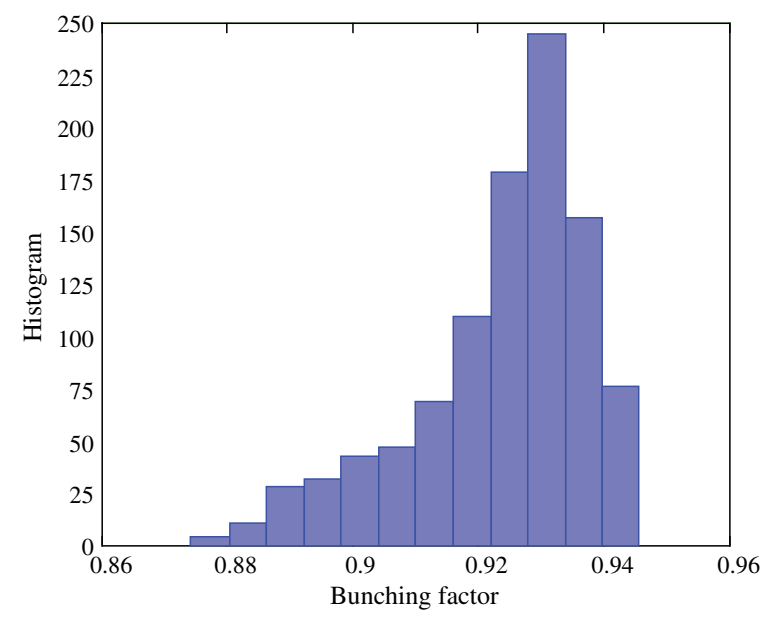

FIG. 8. Histogram of bunching factor results for 1000 different simulations where amplitude and phase for each laser lines vary randomly from their design values by $10 \%$ and 0.5 radians, respectively. gradient accelerators is a very interesting possibility. The output beam has a very narrow phase spread and almost no tails. In general, this scheme shows the flexibility of the IFEL interaction in the manipulation of longitudinal phase space at the optical scale. In fact, the parabolic potential case discussed here is only an example and any desired potential can be expanded and approximated by its Fourier series.

Assuming that a transport line from the buncher to the accelerator entrance would preserve the microbunch structure, we use the longitudinal phase spaces shown in Fig. 7(a) as input of the 3D simulation of the high gradient IFEL accelerator described in the previous section. Results are shown in Fig. 9. A traditional prebuncher significantly improves the performance of the IFEL accelerator, as more particles $(84 \%)$ are captured and accelerated to the final energy than the case of a unbunched beam with a uniform injection phase distribution. In the four harmonic prebuncher case, the fraction of captured particles increases up to $98.5 \%$. But once more the main advantage, especially when considering the IFEL as a potential driver for advanced light sources, is the further reduction of the energy spread which becomes $0.18 \%$. This is due to the fact that the initial phase space has a relatively small area, with
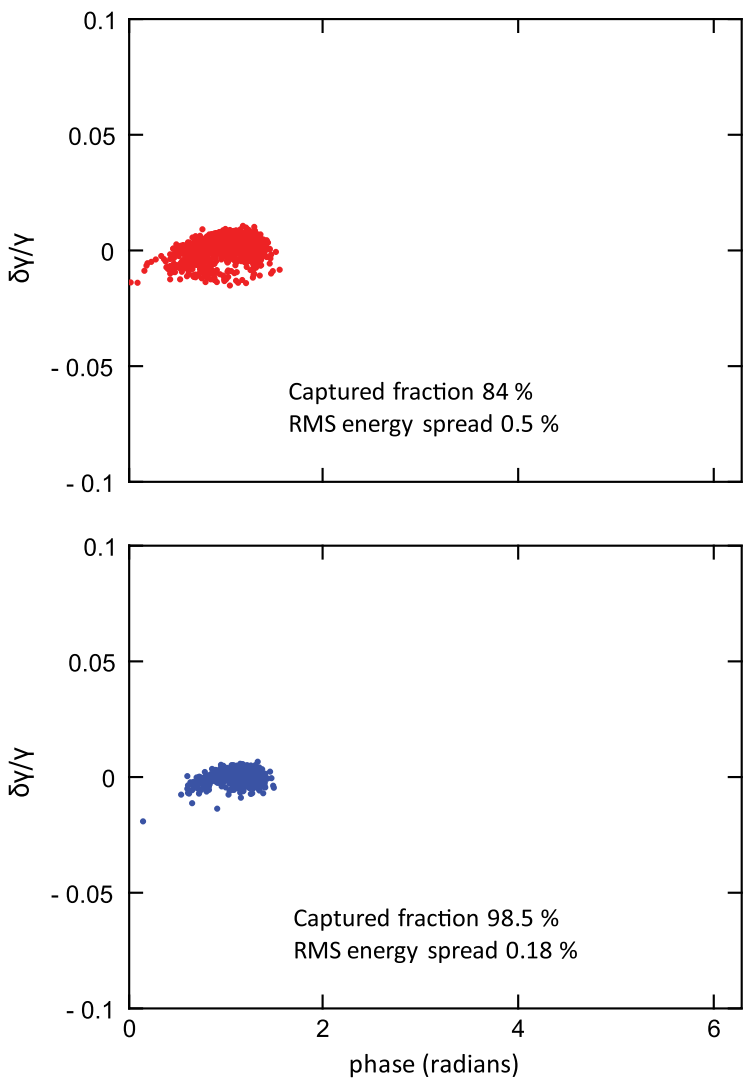

FIG. 9. IFEL output longitudinal phase space and longitudinal distribution for a typical (above) and a harmonic (below) prebuncher. 
a very tight bunch of particles injected at the right phase to experience the high acceleration gradient.

\section{EFFICIENCY OF IFEL BEAM LOADING}

In the description of the IFEL interaction, we have so far considered the electromagnetic radiation as an externally given function rather than as a dynamical variable of the system. This greatly simplifies the modeling of IFEL's and is a very good approximation as long as the accelerated charge is maintained low enough so that effects of beam loading or pump depletion can be neglected. In this section we will analyze the situation when this assumption is no longer valid.

As a first step, it is instructive to look at the energy balance of the system. The energy required to accelerate $1 \mathrm{pC}$ of beam charge to $1 \mathrm{GeV}$ is $1 \mathrm{~mJ}$. This amount of energy needs to be transferred from the laser beam to the electrons. When the fraction of energy absorbed by the beam is significant with respect to the total laser pulse energy, the IFEL dynamics will be affected by the loading of the accelerating gradient from the beam of the accelerating waves.

It is useful to consider a different point of view which gives insight on the mechanism of energy transfer in the undulator. A microbunched electron beam of $1 \mathrm{pC}$ of charge would emit $1 \mathrm{~mJ}$ of coherent radiation at the resonant wavelength when traveling through the IFEL undulator. This radiation is emitted at the resonant wavelength of the undulator but at a phase opposite of the driver laser so that the fields cancel and the radiation effectively loses energy.

In order to describe in a quantitative way the beam loading in an IFEL accelerator, we need to solve the Maxwell equation for the electromagnetic field in presence of a source term consisting in the beam current. The equations are exactly the same as the FEL equations and we can rely on an ample literature of algorithms and codes which have been developed through the years and already well benchmarked with experiments by the FEL community.

For full three-dimensional simulations of the evolution of both the beam as well as the radiation in an IFEL accelerator, we chose one of the most used and benchmarked FEL simulation codes publicly available, GENESIS 1.3 version 2.0 [29]. The code solves the period-averaged equation of motion for the particles and the radiation field equation in the slow-varying envelope approximation. The equation for the longitudinal space charge force electric field can also be solved.

Unfortunately GENESIS cannot be directly used to simulate nonlinearly tapered undulators since the period cannot be changed. On the other hand, tapered undulators are typically designed and built in a piecewise fashion so that each period has a fixed length. Therefore we took advantage of GENESIS' options to import and export a dump of the full 6D phase space of the particles and radiation profiles. Phase space distributions for laser and electrons at the end of each period are fed into a new instance of GENESIS with the undulator vector potential amplitude for the next period.

The simulations could also be run in time dependent mode to simulate the effects of the finite laser pulse and electron bunch lengths. In this case, it is important to shift the radiation slices over the beam slices to take into account the slippage between each period. At the present however, we have not yet implemented this feature.

With this self-consistent simulation tool we can analyze the effects of beam loading in the GeV IFEL design discussed earlier. We take as a reference the undulator design with $\mathrm{NdFeB}$ magnets $\left(B_{r}=1.22 \mathrm{~T}\right)$ and a fixed gap as shown in Fig. 4 with the same input parameters but with the ideally bunched distribution shown in Fig. 9. The accelerator output parameters (final energy and fraction of trapped particles) as a function of input beam current for the case of a prebunched beam are shown in Fig. 10. The final output laser power and beam power gain are also shown.

The final energy of the IFEL does not vary much with beam current since this quantity is determined by the choice of the undulator tapering and not by the dynamics of the system. A significant decrease in the fraction of accelerated particles only begins to occur for very large currents $(>10 \mathrm{kA})$. A reference point is obtained by observing that the power required to accelerate $20 \mathrm{kA}$ of particles to $1 \mathrm{GeV}$ would be $20 \mathrm{TW}$, or the full laser power.
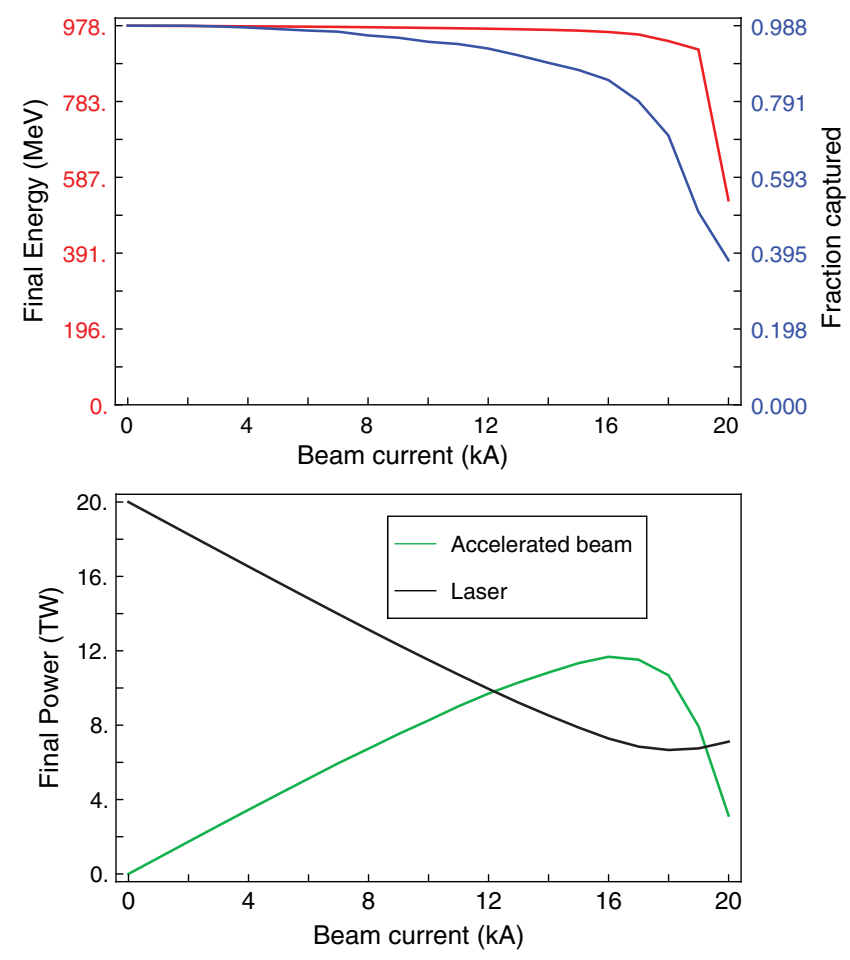

FIG. 10. Output IFEL parameters as functions of input beam current. 
For this example, the maximum power gained by the beam is $11.6 \mathrm{TW}$ so the power transfer efficiency achieved is $58 \%$. The relative energy spread and emittance remained constant. It is also important to realize that the beam loading is dependent on the bunching factor of the injected beam. If the beam is microbunched, the fraction of trapped particles is much larger and the current where the effects of beam loading occur is lower.

For beam currents greater than $18 \mathrm{kA}$ in Fig. 10, the laser loses too much energy along the undulator and the ponderomotive accelerating gradient decreases. The consequence is that the electrons are no longer able to follow the design resonant tapering trajectory and particle detrapping from the stable potential bucket occurs.

Using a simple 1D model, it is possible to optimize the IFEL tapering to take into account the depletion of the IFEL driver and adjust the variation of period and magnetic field amplitude to compensate for the losses in laser power and minimize detrapping.

We can estimate the laser power absorbed in the IFEL when we inject in the accelerator an electron beam of current $I$ since the energy gain is set by the resonance condition,

$$
\begin{aligned}
P_{a b s}(z) & =\eta \operatorname{Im}_{0} c^{2}\left(\gamma(z)-\gamma_{0}\right) \\
& =\eta \operatorname{Im}_{0} c^{2}\left(\sqrt{\lambda_{w}(z)\left(1+K(z)^{2}\right) / 2 \lambda}-\gamma_{0}\right),
\end{aligned}
$$

where $\eta$ is the fraction of particles trapped in the ponderomotive bucket.

By scaling the laser electric field in Eq. (10) as

$$
K_{l}(z) \rightarrow K_{l}(z) \sqrt{\frac{P-P_{a b s}(z)}{P}},
$$

where $P$ is the input laser power, we can find the optimum tapering for a loaded IFEL. The final resonant energy vs design current is shown in Fig. 11. The relaxed tapering towards the undulator's exit allows for acceleration of a larger beam current at the cost of a lower final beam energy.

By using the compensated tapered undulator obtained with this procedure for an example design current of $20 \mathrm{kA}$, we obtain an IFEL design with $>70 \%$ efficiency which improves on the $58 \%$ efficiency of the uncompensated undulator. The results of the simulation for the compensated undulator are shown in Fig. 12. Again we see a nearly constant final energy for various beam currents. The final relative energy spread and emittance are the same as the loaded uncompensated undulator and independent of beam current. In practice, this compensation is not perfect as it assumes that only the trapped particles change energy, and it completely neglects important effects such as harmonic emission, slippage, and three-dimensional effects. Furthermore, these time independent simulations do not account for temporal envelope overlap inefficiencies.

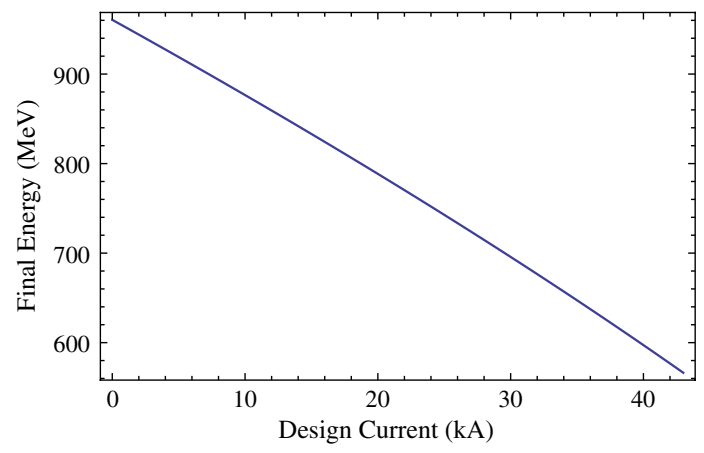

FIG. 11. Final resonant energy versus design beam current for a compensated undulator.

One of the major limitations to the efficiency comes from the fact that the electron beam has a much smaller transverse size than the laser beam in order to experience a nearly constant accelerating gradient. Because of this mismatch, the radiation is not absorbed homogeneously across its transverse cross section.

The laser develops a complex transverse mode as it propagates while an intense electron beam basically burns a hole in the center (see Fig. 13). For small enough beams, the on-axis laser intensity is rapidly depleted, and the electrons slip out of resonance. On the other hand, if the transverse size of the beam is too large, only the fraction of the beam in the region of the laser with sufficient electric field will be accelerated. These competing effects are shown in Fig. 14. In recirculating laser schemes where in order to increase the efficiency the laser power is
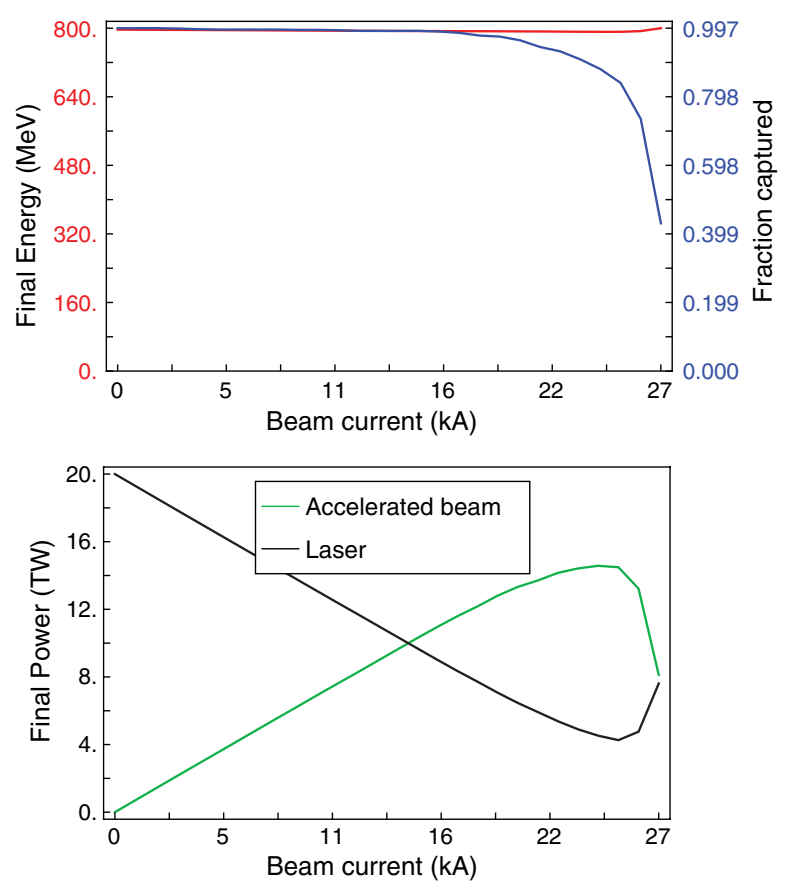

FIG. 12. Output IFEL parameters as functions of input beam current for a compensated tapered undulator designed for $20 \mathrm{kA}$. 

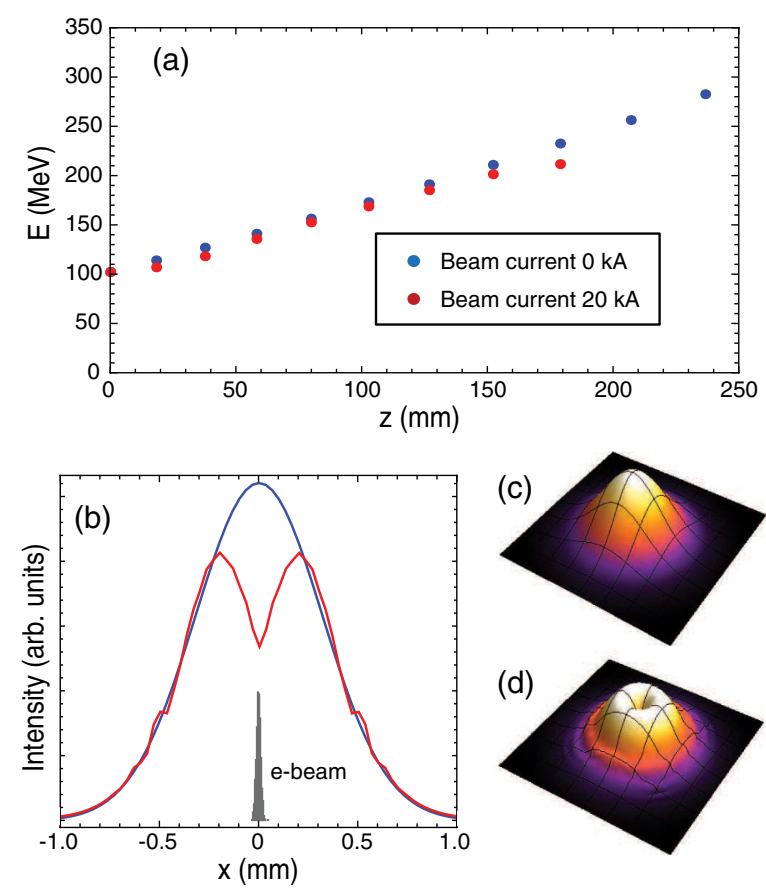

FIG. 13. (a) Mean beam energy vs position along undulator for a $0 \mathrm{kA}$ and $20 \mathrm{kA}$ beam. The $20 \mathrm{kA}$ beam slips from resonance by the 9 th undulator period. (b) Laser profiles at period 8 for the $0 \mathrm{kA}$ and $20 \mathrm{kA}$ beams (electron beam distribution shown in gray). (c) Laser intensity profile for the $0 \mathrm{kA}$ beam at the 8th period. (d) Laser intensity profile for the $20 \mathrm{kA}$ beam at the $8 \mathrm{th}$ period.

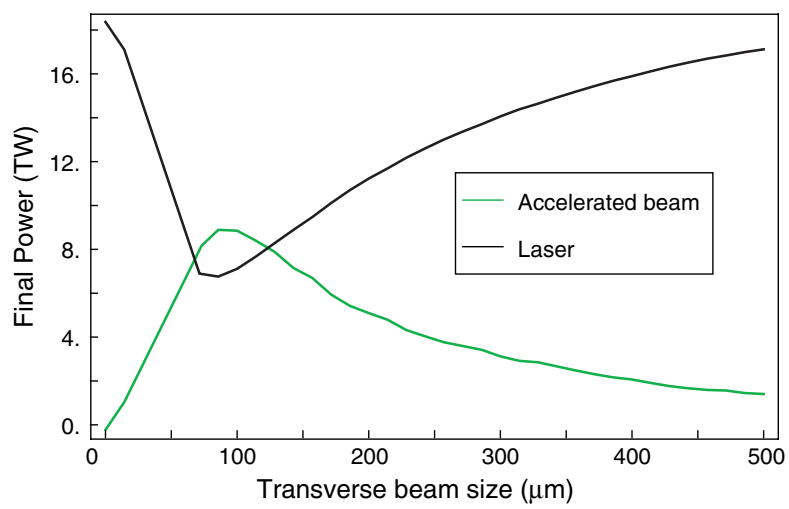

FIG. 14. Power absorbed by accelerated electrons and remaining laser power vs rms transverse beam size for the case of the uncompensated undulator and a $18 \mathrm{kA}$ beam.

replenished by a laser amplifier, it will be important to introduce optical elements in the recirculating cavity to restore the transverse profile before reusing the power for IFEL acceleration.

\section{CONCLUSION}

IFEL acceleration schemes enjoy a period of rejuvenated interest due the feasibility of $1-2 \mathrm{GeV}$ compact IFEL injectors for light source applications. These include soft$\mathrm{x}$-ray FEL amplifier and gamma-ray generation by inverse Compton scattering [11,12]. At the moment, the control on the beam quality offered by the IFEL does not have equal among other laser acceleration schemes. For this reason, IFEL is the best candidate to produce the most suitable beams for advanced light source applications. In the next few years, IFEL experiments at BNL and LLNL will pave the way towards the design of an advanced light source based on the IFEL acceleration scheme [13,14]. The continuous progress of the laser technology has made terawatt tabletop systems available to a smaller scale university size laboratory. In this paper it is shown that an IFEL accelerator driven by a 20 TW laser system can accelerate preserving the emittance to a $1 \mathrm{GeV}$ beam with $<0.5 \mathrm{~mm}$ mrad normalized emittance, $>1 \mathrm{kA}$ in current in $1 \mathrm{~m}$. The final energy spread can be smaller than $0.2 \%$. One issue for the IFEL scheme is that the input electron energy still needs to be quite high $(100 \mathrm{MeV}$ in our example); however, novel undulators with $\mathrm{mm}$ period, or the exploitation of the IFEL harmonic interaction could offer a solution to this problem and further shrink the size of IFEL based light sources.

\section{ACKNOWLEDGMENTS}

This work was supported by DOE Grant No. DE-FG0292ER40693, Defense of Threat Reduction Agency Award No. HDTRA1-10-1-0073, and University of California Office of the President Award No. 09-LR-04-117055MUSP.

[1] Y. Liu, X. J. Wang, D. B. Cline, M. Babzien, J. M. Fang, J. Gallardo, K. Kusche, I. Pogorelsky, J. Skaritka, and A. van Steenbergen, Phys. Rev. Lett. 80, 4418 (1998).

[2] Z. Huang, M. Borland, P. Emma, J. Wu, C. Limborg, G. Stupakov, and J. Welch, Phys. Rev. ST Accel. Beams 7, 074401 (2004).

[3] A. Mikhailichenko and M. Zolotorev, Phys. Rev. Lett. 71, 4146 (1993).

[4] C. M.S. Sears et al., Phys. Rev. ST Accel. Beams 11, 101301 (2008).

[5] L. H. Yu et al., Science 289, 932 (2000).

[6] A. Zholents, Phys. Rev. ST Accel. Beams 8, 040701 (2005).

[7] R. Palmer, J. Appl. Phys. 43, 3014 (1972).

[8] E. D. Courant, C. Pellegrini, and W. Zakowicz, Phys. Rev. A 32, 2813 (1985).

[9] W. Kimura et al., Phys. Rev. Lett. 92, 054801 (2004).

[10] P. Musumeci et al., Phys. Rev. Lett. 94, 154801 (2005).

[11] P. Musumeci and M. Serluca, in Proceedings of the 2006 FEL Conference, Berlin, Germany, 2006 [http://www .jacow.org/].

[12] A. Tremaine et al., in Proceedings of the 2011 Particle Accelerator Conference, New York, NY, USA (2011). 
[13] J.P. Duris et al., in Proceedings of the 2011 Particle Accelerator Conference, New York, NY, USA (2011).

[14] S. G. Anderson et al., in Proceedings of the 2011 Particle Accelerator Conference, New York, NY, USA (2011).

[15] N. M. Kroll, P. L. Morton, and M. N. Rosenbluth, IEEE J. Quantum Electron. 17, 1436 (1981).

[16] W. Zakowicz, J. Appl. Phys. 55, 3421 (1984).

[17] R. Tikhoplav et al., in Proceedings of the 8th European Particle Accelerator Conference, Paris, 2002 (EPS-IGA and CERN, Geneva, 2002).

[18] Amplitude Technologies, 2-4 rue du Bois Chaland, CE 2926, 91029 EVRY, France [http://www.amplitudetechnologies.com/].

[19] K. Halbach, Nucl. Instrum. Methods 187, 109 (1981).

[20] F. H. O'Shea, G. Marcus, J. B. Rosenzweig, M. Scheer, J. Bahrdt, R. Weingartner, A. Gaupp, and F. Gruner, Phys. Rev. ST Accel. Beams 13, 070702 (2010).

[21] Kaan Uestuener, Matthias Katter, Rolf Blank, Daniela Benedikt, Johannes Bahrdt, Andreas Gaupp, Bastian Klemke, Florian Grüner, and Raphael Weingartner, in Proceedings of the 20th International Workshop on Rare Earth Permanent Magnets and their Applications, Knossos-Crete, Greece, 2008, edited by D. Niarchos (Admore, Greece, 2008).
[22] Christopher M.S. Sears, Eric R. Colby, Benjamin M. Cowan, Robert H. Siemann, and James E. Spencer, Phys. Rev. Lett. 95, 194801 (2005).

[23] P. Musumeci, C. Pellegrini, and J. B. Rosenzweig. Phys. Rev. E 72, 016501 (2005).

[24] S. Ya. Tochitsky, O. B. Williams, P. Musumeci, C. Sung, D. J. Haberberger, A. M. Cook, J. B. Rosenzweig, and C. Joshi, Phys. Rev. ST Accel. Beams 12, 050703 (2009).

[25] S. Pottorf and X. J. Wang, in 18th Advanced ICFA Beam Dynamics Workshop on Quantum Aspects of Beam Physics, edited by P. Chen (World Scientific Publishing Co., Singapore, 2002), p. 232.

[26] Han-Sung Chan, Zhi-Ming Hsieh, Wei-Hong Liang, A. H. Kung, Chao-Kuei Lee, Chien-Jen Lai, Ru-Pin Pan, and Lung-Han Peng, Science 331, 1165 (2011).

[27] S. N. Goda, M. Y. Shverdin, D. R. Walker, and S. E. Harris, Opt. Lett. 30, 1222 (2005).

[28] D. F. Ratner and A. W. Chao, Phys. Rev. Lett. 105, 154801 (2010).

[29] S. Reiche, K. Goldhammer, and P. Musumeci, in Proceedings of PACO7 (IEEE, Albuquerque, New Mexico, 2007). 